\title{
La psicología en Guatemala, un poco de historia y actualidad
}

\section{Psychology in Guatemala, some of its history and present}

Fecha de recepción: 07/04/2015

Fecha de aceptación: 18/06/2015

\author{
M.Sc Alma Guisela Cárcamo Duarte \\ M.A Ana Cecilia Escobar Martínez
}

\section{resumen/alsstract:}

Una de cada cuatro personas entre 18 y 65 años en Guatemala, refiere sufrir o haber sufrido alguna vez en su vida un trastorno mental. La atención de la salud mental en el país constituye un reto, más aún cuando menos del 1\% del presupuesto nacional en salud es destinado para ello. No obstante, Guatemala ha logrado avances importantes en cuanto a formación de recurso humano y ejercicio de la psicología se refiere. El país cuenta con catorce universidades, trece son privadas y una estatal, existiendo programa de psicología en once de ellas. Esto ofrece la posibilidad de formación a través de programas técnicos y profesionales, tanto de pregrado como a nivel de especialización. La organización gremial se ha desarrollado a través de asociaciones profesionales y recientemente, en el 2007, con la constitución del Colegio de Psicólogos de Guatemala. Actualmente, los psicólogos realizan aportes desde diferentes espacios y sectores, más allá de la clínica privada y el sector académico, como tradicionalmente se daba. Existen retos importantes y vacíos por cubrir, como el desarrollo de la investigación, la mejora de la calidad en la formación académica y la proyección a los sectores en condición de mayor vulnerabilidad en el país.

One in four people aged 18 to 65 years in Guatemala, suffers or have ever suffered in his life a mental disorder. Mental health care is a real challenge in this country, especially when less than $1 \%$ of the national health budget is allocated for it. However, Guatemala has made important advances in human resource training and practice of psychology. The country has fourteen universities, thirteen are private and one is public, and eleven of them have a psychology program. This offers the possibility of training through technical and professional programs, both at an undergraduate and a specialization level. The trade union organization has been developed through professional associations and recently, in 2007, with the establishment of the Guatemala's Official College of Psychologists. Currently, psychologists make contributions from different areas, beyond the private practice and academia, as traditionally has been done. Still, there are important challenges and gaps to be addressed, such as research development, improving the quality of academic education and social projection to those sectors in most vulnerable conditions.

\section{palabras clave/keywords:}

Psicología, asociación gremial, formación, Guatemala

Psychology, academic education, trade union, Guatemala.

La Encuesta Nacional de Salud Mental, realizada en el 2009 por el Centro de Investigaciones de las Ciencias de la Salud (2010) de la Universidad de San Carlos en colaboración con otras instituciones, constituye el único estudio epidemiológico a nivel nacional sobre la prevalencia de trastornos mentales en el país y el primer estudio de su tipo también en Centroamérica. Los resultados muestran que uno de cada cuatro guatemaltecos, entre 18 y 65 años, ha padecido al menos un trastorno de salud mental en su vida. En términos generales 
los trastornos de ansiedad constituyen el grupo de trastornos más frecuente que afecta a la población guatemalteca (20.6\%), en el cual la más alta prevalencia la tiene el trastorno de estrés post-traumático con un $6.9 \%$ de los cuales solamente el $2.3 \%$ ha buscado atención por un profesional de la salud.

El Instrumento de Evaluación para Sistemas de Salud Mental de la Organización Mundial de la Salud, constituye una herramienta para recopilar la información esencial sobre el sistema de salud mental de un país o región. Guatemala desarrolló el primer ejercicio en el 2006 y finalizó la segunda versión en el 2010. La información de esta evaluación, indica que no ha habido cambios en la asignación presupuestaria para la atención en salud mental, menos del $1 \%$ de los egresos en atención de salud están dirigidos a la atención en salud mental por parte del Ministerio de Salud Pública y Asistencia Social y de este porcentaje el 94\% está dirigido al hospital psiquiátrico. La atención que brindan los establecimientos de salud mental ambulatorios disponibles en el país, es de aproximadamente 363 usuarios por cada 100,000 habitantes. Los resultados también muestran que el 58\% de los usuarios son mujeres y $14 \%$ niños o adolescentes, los principales diagnósticos son trastornos neuróticos y somatomorfos (54\%) y trastornos afectivos (24\%). Guatemala cuenta con un total de tres hospitales psiquiátricos, uno de ellos privado (Organización Mundial de la Salud, 2011).

Los datos evidencian la necesidad de disminuir la brecha de atención en salud mental para la población guatemalteca e integrar la atención en los servicios de atención primaria, estrategias priorizadas en el Plan Estratégico Nacional para el fomento de la salud mental en Guatemala 2007-2020 (MSPAS, 2008a) publicado por el Ministerio de Salud Pública y Asistencia Social en el 2008. En ese mismo año se publicó la Política Nacional de Salud Mental, que busca integrar el componente de salud mental en el modelo de salud integral, fortalecer la capacidad técnica a nivel comunitario e intervenir sobre los determinantes sociales que inciden sobre ésta, a la vez que se promueven estilos de vida saludables y se desarrollan acciones de vigilancia e investigación que faciliten la toma de decisiones orientadas a ofrecer atención oportuna (MSPAS, 2008b). El país no cuenta con legislación específica sobre salud mental, pero afortunadamente este es un tema sobre el que ya se trabaja desde el 2013 y del que existen esfuerzos previos.

En cuanto a la formación del recurso humano, Guatemala cuenta actualmente con catorce universidades (trece privadas y una estatal) existiendo programa de psicología en once de ellas (San Carlos, Rafael Landívar, del Valle de Guatemala, Francisco Marroquín, Mariano Gálvez, Galileo, del Istmo, Mesoamericana, Internaciones, San Pablo y Rural). La carrera de psicología nace al interior de la Facultad de Humanidades de la Universidad de San Carlos, única universidad pública del país, en el año 1946, contando únicamente con la licenciatura en Psicología y Profesorado de Enseñanza Media en Psicología (Aguilar y Recinos, 1996). El 23 de julio de 1974, producto de un movimiento estudiantil generado por las constantes demandas por una reestructura académica sustancial del Departamento de Psicología, éste se desliga de la Facultad de Humanidades, constituyéndose, según Acuerdo del Consejo Superior Universitario de esa casa de estudios superiores, de fecha 24 de julio de 1974, en la Escuela de Ciencias Psicológicas, que hoy día se encuentra ubicada en el Centro Universitario Metropolitano. En conmemoración a esa gesta estudiantil, según

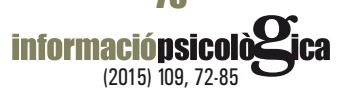


Acuerdo Gubernativo Número 1385-90 de fecha 28 de diciembre de 1990, el Lic. Marco Vinicio Cerezo Arévalo, Presidente de la República, acuerda declarar el 23 de julio de cada año, como el "Día del Psicólogo".

La Escuela de Ciencias Psicológicas de la Universidad de San Carlos inicia operaciones con un plan piloto de pensum de estudios, aprobando en 1976 tres niveles académicos: el nivel técnico, el nivel de licenciatura y el nivel de postgrados, éste último se concretó años después; pudiéndose obtener los títulos de profesorado en orientación vocacional, laboral y escolar; terapia del lenguaje; educación especial en enseñanza media en psicología; terapia ocupacional y recreativa; y, licenciatura en psicología. Según refieren Aguilar y Recinos (1996), el método de enseñanza aprendizaje era de unidades integradoras, que en 1981 desaparece para dar paso al régimen anual de estudios, en el que los estudiantes cursan cinco asignaturas en cada grado, más investigación y práctica profesional. A nivel de postgrado, se inician la maestría en Psicología Social y el Profesorado en Enseñanza Superior en Metodología Integral, mismas que no prosperaron (Aguilar y Recinos). En 1989 el Consejo Superior Universitario aprueba la creación de la Escuela de Ciencia y Tecnología de la Actividad Física y el Deporte (ECTAFIDE), adscrita a la Escuela de Ciencias Psicológicas, según punto séptimo del acta 67-89 de fecha 25 de octubre de 1989. Posteriormente, según punto vigésimo, acta 26 de fecha 15 de noviembre de 1996 de ese mismo órgano de dirección, ECTAFIDE pasa a funcionar en calidad de carrera de la Escuela de Ciencias Psicológicas.

Según lo indica el licenciado Abraham Cortez Mejía (comunicación personal, 28 de julio de 2014), director de la Escuela de Ciencias Psicológicas de la Universidad de San Carlos, fue en 1997 que se inicia la reestructura curricular, la que por situaciones muy particulares de la Escuela, se implementa hasta el 2010, cambiando de régimen anual a régimen semestral, que es como funciona actualmente.

El programa de Práctica Supervisada se inició en 1976 (Aguilar y Recinos 1996), el cual a través de los años ha sido fortalecido. Los estudiantes pueden realizar práctica en las áreas clínica, educativa, industrial y social comunitaria, teniendo mayor demanda las áreas clínica y educativa. Los estudiantes de noveno y décimo semestre eligen un electivo según el área del conocimiento y el centro de práctica, de acuerdo a su promedio de notas (A. Cortez Mejía, comunicación personal, 28 de julio de 2014). Las prácticas se llevan a cabo en la red de servicios de salud del país, en organizaciones no gubernamentales, asociaciones benéficas y centros educativos.

A nivel de postgrado, la Escuela de Ciencias Psicológicas ofertó la maestría en Psicología Social y Violencia Política, la que después de algunos años, por problemas administrativos, fue cancelada, según se hace constar en acta de la sesión extraordinaria del Consejo Directivo de la Escuela de Ciencias Psicológicas, celebrada el 28 de septiembre de 2009. A la fecha se imparte la maestría en Psicología forense, proyectando iniciar la maestría en Análisis social de la discapacidad en el año 2015 y se tiene en proyecto la maestría en Psicología industrial. A nivel de especializaciones, en coordinación con el departamento de neurología del Hospital San Juan de Dios, se cuenta con el curso de especialización en Neuropsicología. Para julio 2015 se contempla iniciar los cursos de actualización en

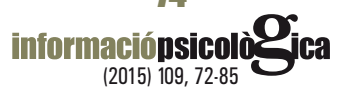


Evaluación para la selección de personal y para agosto del mismo año, el curso Evaluación neuropsicológica del adulto. Es importante mencionar que en 2002 se inició la maestría y doctorado en Psicología, programa que fue cerrado en 2013 por problemas administrativos, según acta cinco/2013 de la sesión ordinaria del Consejo Directivo del Sistema de Estudios de Postgrado. Si bien no hubo graduados de estos programas, el licenciado Cortez refiere que se hacen los esfuerzos necesarios para graduar a los estudiantes con cierre de pensum (comunicación personal, 28 de julio de 2014).

La Universidad de San Carlos ha descentralizado la educación pública superior, contando con centros universitarios en 20 de los 22 departamentos del país, en algunos se ha implementado la licenciatura en Psicología, por lo que las autoridades de la Escuela de Ciencias Psicológicas están trabajando en la homologación de la carrera.

Los títulos académicos que actualmente ofrece la Escuela de Ciencias Psicológicas a nivel técnico son: Profesor de enseñanza media en psicología, Orientador vocacional y laboral, Terapista ocupacional y recreativo, Terapista del lenguaje, Profesor en educación especial, Técnico en deportes, profesorado de enseñanza media en educación física y Técnico en recreación. A nivel de licenciatura: Licenciado en psicología y Licenciado en educación física, deporte y recreación. En la actualidad es el programa de psicología que más estudiantes acoge y gradúa.

Quince años después de abrirse la carrera de Psicología en la Universidad de San Carlos, se funda la Universidad Rafael Landívar, la que incluye, desde su creación, el Departamento de Psicología adscrito a la Facultad de Humanidades, facultad a la que pertenece a la fecha, y que ofrece la carrera de licenciatura en psicología. En 1975 se crea el doctorado en psicología clínica y escolar, graduando únicamente a dos profesionales, por lo que se procede a cerrar dicho programa (Aguilar y Recinos 1996).

Según Aguilar y Recinos (1996), el Departamento de psicología, en el año de 1977, implementó las carreras técnicas de Orientación escolar, Psicometría, Educación especial para retardados mentales (término utilizado en esa época) y Problemas del lenguaje; luego para 1977 ofrecía las carreras de licenciatura en Administración de recursos humanos, Psicología educativa y Psicología clínica. Refiere la licenciada Georgina Mariscal de Jurado (comunicación personal, 24 de julio de 2014), actual directora del Departamento de psicología, que en 1995 debido a una reestructuración académica, se crean las licenciaturas en Psicología educativa (a partir de 2012 ya no se oferta), Psicología clínica y Psicología industrial/ organizacional y la carrera técnica en Audición y lenguaje. A nivel de postgrado ofertan las maestrías en Gestión y desarrollo estratégicos del talento humano, en Gestión del desarrollo de la niñez y adolescencia y la maestría en Psicología clínica y prácticas psicoterapéuticas.

En cuanto al área de práctica, cuenta con el Centro Landivariano de Práctica de Psicología, donde estudiantes de los últimos años de la carrera de licenciatura en Psicología clínica realizan su práctica supervisada, aunque también pueden ser ubicados en otros centros para realizarla. La práctica de la carrera de Psicología industrial/organizacional se realiza en empresas privadas. Las jornadas de estudios son matutina y vespertina, ambas carreras tienen una duración de cinco años. (G. Mariscal de Jurado, comunicación personal, 24 de julio de 2014).

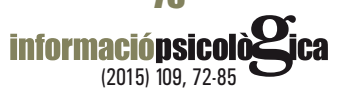


La Universidad Rafael Landivar también ha descentralizado sus servicios, cuenta con sedes en diferentes departamentos del país, ofertando en algunos de ellos la carrera de licenciatura en Psicología industrial/organizacional y de Psicología clínica.

La Universidad del Valle de Guatemala ha hecho importantes aportes para el desarrollo de la psicología guatemalteca, específicamente en el área psicométrica (Aguilar y Recinos, 1996). En 1951 el Colegio Americano de Guatemala fundó la Oficina de Investigación, con el Servicio Cooperativo Interamericano de Educación, generando la elaboración de pruebas psicométricas en español y priorizando la adaptación de los test de Habilidad General, test de Aptitud Diferencial y pruebas de lectura. Una de las primeras estandarizaciones realizadas fue la del Otis Intermedio y Superior Forma A. La Oficina de Investigación estuvo bajo el cargo del Dr. Otto E. Gilbert Almengor prominente psicólogo guatemalteco.

En 1956, a través del Colegio Americano, se proporciona al Servicio Cooperativo Interamericano de Educación el test Pitner Cunningham, forma A, traducido y adaptado al medio guatemalteco. Con los datos obtenidos, posteriormente se elaboraron las normas para niños guatemaltecos del test de Habilidad General Pitner Cunningham para párvulos, preparatoria y primer grado de primaria. Entre 1954 y 1961 se tradujo los test de Aptitud Diferencial (TAD), se elaboró el test de Lectura de Nivel Intermedio y posteriormente se crearon los test de Aptitud Forma H (Aguilar y Recinos, 1996).

Al fundarse la Universidad del Valle de Guatemala en 1966, la Oficina de Investigación del Colegio Americano pasa a constituirse como el Centro de Investigaciones Educativas de la Universidad del Valle de Guatemala, quedando oficialmente adscrito al Instituto de Investigaciones en el año 1986 (Aguilar y Recinos, 1996).

El Departamento de Psicología, es fundado en el año de 1976, como parte de la Facultad de Ciencias y Humanidades (Aguilar y Recinos, 1996), en la actualidad pertenece a la Facultad de Ciencias Sociales.

Según información dada por el licenciado Pablo Barrientos Marroquín, Director del Departamento de Psicología (comunicación personal, 17 de julio de 2014), el nivel de pregrado consta de cinco años de formación general. Los cursos se dividen en cursos de formación general, de ciencia básica de la profesión y de formación profesional. La práctica supervisada tiene una duración de 900 horas y se inicia en el segundo semestre del cuarto año de la carrera y finaliza en el quinto año., número de horas que ha aumentado significativamente, pues en los inicios de la carrera eran únicamente 80 horas (Aguilar y Recinos 1996).

Para graduarse el estudiante debe realizar un trabajo de tesis, la carrera brinda al estudiante la oportunidad de integrar la teoría, práctica e investigación de los fundamentos del comportamiento humano, del individuo y de su grupo social. Su plan de estudios sigue criterios de acreditación nacionales e internacionales y forma parte de la Red Iberoamericana de Facultades de Psicología (P. Barrientos Marroquín, comunicación personal, 17 de julio de 2014).

A nivel de post grado el Departamento de Psicología ofrece las maestrías en Gestión del talento humano y en Consejería psicológica y salud mental, dirigidas por la Dra. Grazioso de Rodríguez destacada profesional guatemalteca, quien ha promovido el desarrollo de la 
psicología en Guatemala y ha tenido una reconocida participación como miembro de la Sociedad Interamericana de Psicología (SIP). Este programa ofrece un sistema de módulos, impartidos por profesores con grado académico de doctorado y especialización en su área de docencia. Previo a graduarse los estudiantes deben haber aprobado 18 módulos, una práctica clínica supervisada, un internado comunitario, un portafolio, una publicación o presentación en congreso y un trabajo de investigación cualitativo. Asimismo, se les sugiere seguir un proceso psicoterapéutico personal paralelo a su formación teórica, profesional y científica (P. Grazioso, comunicación verbal, 18 de julio de 2014).

El Departamento de Psicología de la Universidad Mariano Gálvez, hoy Facultad de Psicología, nace en el año de 1986 (Aguilar y Recinos 1996). Al igual que en otras universidades la carrera tiene cinco años de duración, y su orientación ha sido hacia el área clínica. A diferencia de otros programas de psicología, el de la Universidad Mariano Gálvez se ha caracterizado por ser dirigido por psiquiatras, en la actualidad el decano de la facultad es el neurólogo Carlos Ramírez Monterrosa.

Refiere Beatriz Menchú, que para poder graduarse los alumnos en este programa deben cumplir con 200 horas de práctica y después de cerrar pensum deben elaborar un trabajo de tesis. Actualmente, la Facultad de Psicología de la Universidad Mariano Gálvez ofrece las carreras de técnico universitario en Psicología y técnico universitario en Educación especial, así como las licenciaturas en Psicología, Psicología educativa, Psicología clínica y Psicología industrial.

Los programas de postgrado inician en el año de 1986, se imparten a través de la Escuela Superior de Postgrado de Dinámica Humana y Salud Mental, contando con el aval académico de la Universidad Mariano Gálvez, desde sus inicios han estado a cargo del Dr. Cyrano Ruiz Herrarte, psiquiatra guatemalteco ("Escuela Superior de Postgrado de Dinámica Humana y Salud Mental", 2014). Las carreras del programa de postgrado se agrupan en dos áreas: clínicas y no clínicas y en todas ellas los grados académicos son: maestría, doctorado y post doctorado. Los postgrados que ofrecen son: Psicología clínica y salud mental, Psicología médica y salud mental, Psiquiatría de adultos y salud mental, Psiquiatría de niños y salud mental y, Dinámica humana y salud mental.

En el año de 1990 la oferta de los programas de postgrado se extiende hacia el suroccidente del país, funcionando actualmente una extensión en la ciudad de Quetzaltenango.

Al contrario que en otras universidades, la Universidad Francisco Marroquín inicia con programas a nivel de post grado, esto gracias a la iniciativa del Dr. Guillermo Forno, psiquiatra guatemalteco (Aguilar y Recinos 1996). Es en 1975, inicia actividades la Escuela Superior de Psicología Clínica y Médica, a través del Instituto de Ciencias de la Conducta, con el aval académico de esa casa de estudios, impulsando las maestrías y doctorados en Psicología clínica y médica. Por problemas personales el Dr. Guillermo Forno se ve obligado a salir del país, quedando los programas a cargo de la Universidad Francisco Marroquín, los que posteriormente dejan de funcionar (Aguilar y Recinos, 1996). La oferta académica de la carrera de licenciatura en Psicología se lanza en el año de 1978 (Aguilar y Recinos, 1996), un año más tarde se crea la licenciatura en Psicología industrial y un técnico en Recursos humanos. En 1981 se crea la maestría en Psicología industrial.

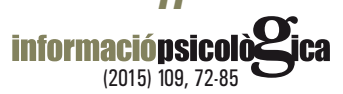


Aguilar y Recinos (1996) refieren que en 1984 asume la dirección del Departamento de Psicología el Dr. Luis Recinos, quien reestructura el departamento, ofreciendo la licenciatura en Psicología clínica, una maestría en Administración de recursos humanos y un técnico en Administración de personal. Hacia 1993, nace la licenciatura en Psicología Industrial.

Inicialmente los estudiantes de la carrera de licenciatura en Psicología clínica realizaban sus prácticas en el Hospital Nacional de Salud Mental (hospital público) y en el Hospital Hermano Pedro (hospital privado), por razones de espacio, decide trasladar la atención de pacientes de consulta externa directamente a la universidad. En 1994, se fundan las clínicas de psicología, hoy Centro de Práctica Clínica Viktor Frankl donde realizan su práctica supervisada (Aguilar y Recinos 1996).

El departamento de Psicología de la Universidad Francisco Marroquín ha generado importantes contribuciones sobre la psicología del niño y adolescente guatemalteco (Aguilar y Recinos 1996), esto a través de investigaciones financiadas por el Fondo Conmemorativo Charles L. Stillman, fondo destinado a la capacitación y actualización profesional de sus docentes, así como para la realización de investigaciones y publicaciones.

Actualmente la Universidad Francisco Marroquín ofrece las carreras de licenciatura en Psicología clínica, licenciatura en Psicología empresarial, ambas en plan diario, la licenciatura en Recursos humanos, así como un técnico en Administración de personal en plan sábado, teniendo los estudiantes la posibilidad de, al concluir el técnico, continuar con la licenciatura en Recursos humanos.

Según información recabada en el Departamento de Psicología, en las carreras de licenciatura, los alumnos deben cumplir con 600 horas de práctica supervisada, los estudiantes de la licenciatura en Psicología Clínica realizan su práctica en el Centro de Práctica Clínica Viktor Frankl y los de la licenciatura en Psicología Empresarial en empresas del sector privado. La directora actual del departamento de Psicología es la Dra. Yetilú Iunge de Baessa (Departamento de Psicología, comunicación personal, 2 de agosto de 2014).

El Departamento de Psicología ofrece la maestría en Neurofisiología del comportamiento, que es un área de estudios nueva, que permite al graduado trabajar en equipo con médicos, psiquiatras y nutricionistas, entre otros. El programa tiene una duración de dos años, incluyendo la tesis y la práctica hospitalaria, la que se realiza en un hospital privado (Hospital Universitario Esperanza). Su objetivo es proporcionar al estudiante una formación científica y técnica, altamente calificada que lo capacite para el correcto ejercicio multidisciplinario en el contexto del trabajo de la psicología ("Departamento de Psicología”, 2014).

Por su parte, la Universidad Galileo da aval académico a los programas de psicología que oferta el Instituto de Ciencias de la Familia, dirigido por la licenciada María de los Ángeles Figueroa de Mollinedo. Ofrecen las carreras de licenciatura en Psicología clínica y licenciatura en Psicología familiar con especialidad en logoterapia. Asimismo ofrecen maestrías en Logoterapia y en Psicoterapia familiar y conyugal ("Instituto de Ciencias de la Familia", 2014). 
La Universidad del Istmo, cuenta con la licenciatura en Psicología clínica y licenciatura en Psicopedagogía clínica, carreras que pertenecen a la Facultad de Educación, tiene una duración de cuatro años, los estudiantes realizan una práctica clínica de 400 horas por semestre desde el primer año, llevando casos a los que se les da seguimiento durante los cuatro años de la carrera y que al momento del cierre de pensum sirven para la elaboración de la tesis de graduación, (Facultad de Educación, comunicación personal, 26 de julio de 2014).

Por su parte la Universidad Da Vinci crea la Facultad de Ciencias de la Salud en el año 2013, ofertando las carreras de licenciatura en Psicología Clínica, Educativa e Industrial/ Organizacional. La Universidad Da Vinci tiene su sede en el Departamento de Huehuetenango, en el noroccidente del país. ("Facultad de Ciencias de la Salud", 2014)

La Universidad Panamericana cuenta con la Facultad de Ciencias Psicológicas, ofreciendo las carreras de licenciatura en Psicología organizacional y gestión del talento humano, licenciatura en Psicología clínica y consejería social, teniendo tres diplomados de salida, a los dos años Diplomado en Intervención psicológica preventiva, a los tres años, Diplomado en orientación vocacional y a los cuatro años, Técnico en consejería familiar y social; y licenciatura en Psicología educativa, la que tiene tres técnicos de salida, a los dos años, Diplomado en coaching y aprendizaje, al tercer año, Profesorado en pedagogía y psicología y al cuarto año, técnico universitario en Atención a capacidades educativas especiales. Los programas de licenciatura tienen una duración de cinco años, con modalidad trimestral, (se imparten en plan diario y fin de semana), a su egreso los estudiantes tienen que haber cumplido con la práctica profesional dirigida, sistematización de la práctica profesional dirigida, informe técnico de práctica y el examen técnico profesional. Así también, oferta la maestría en Consejería Clínica e Intervención Psicosocial en Salud Mental ("Facultad de Ciencias Psicológicas, 2014).

La Universidad San Pablo ofrece la carrera de licenciatura en Psicología Industrial y Comportamiento Organizacional, que pertenece a la Escuela de Liderazgo y Empresarialidad, se imparte en plan semanal en jornada nocturna y en plan fin de semana, la carrera tiene una duración de cuatro años ("Escuela de Liderazgo y Empresarialidad", 2014).

Por su parte, la Universidad Internaciones a través de la Escuela de Psicopedagogía y Educación perteneciente a la Facultad de Humanidades, oferta las carreras de licenciatura en Psicología con especialización en Psicología Educativa, licenciatura en Psicología con especialización en Psicología Empresarial y licenciatura en Psicología con especialización en Psicología Social. Las carreras tienen una duración de cinco años, con modalidad semestral (“Escuela de Psicopedagogía y Educación”, 2014).

Como evaluaciones de contenidos se recurre a exámenes parciales y finales, y para acumular zona se asignan trabajos de investigación individuales y de grupo. Previo a graduarse deben realizar práctica supervisada, posteriormente deben elaborar una tesis o informe del Ejercicio Profesional Supervisado, EPS y sustentar un examen público. En la carrera de Psicología Industrial de la Escuela de Ciencias Psicológicas de la Universidad de San Carlos, refiere su fundador, licenciado Mariano Codoñer Castillo, cada cinco años se lleva a cabo una investigación de campo para evaluar el impacto de la carrera en el mercado

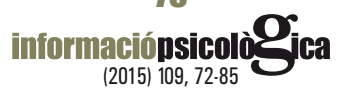


laboral (M. Codoñer, comunicación personal cinco de julio de 2014). En los programas de postgrados, además de los requisitos ya citados, el estudiante debe sustentar exámenes privados. El cuerpo de profesores, especialmente en maestrías y doctorados, son profesionales de reconocido prestigio y algunos son profesores invitados de otros países. La evaluación de la calidad académica también incluye evaluaciones periódicas a docentes, autoridades y programas para asegurar que los niveles de formación y entrenamiento sean los pertinentes.

Al graduarse, para poder ejercer legalmente la profesión, los psicólogos deben colegiarse en el Colegio de Psicólogos de Guatemala, según lo establece la Ley de Colegiación Profesional Obligatoria (Decreto 72-2001), status que como valor agregado promueve la organización gremial. Por varios años, psicólogos ávidos de que existiera un cuerpo colegiado que los agremiara y velara por su bienestar y que promoviera el desarrollo de la psicología en Guatemala, hicieron el intento de fundar asociaciones de psicología y un colegio profesional; sin embargo esos esfuerzos fueron infructuosos.

Fue hasta el año de 1996 que un grupo de profesionales liderado por la maestra Ana María Jurado tuvo la iniciativa de fundar la Asociación Guatemalteca de Psicología (AGP). Es así como nace la primera institución con personería jurídica que reuniría a profesionales de una misma disciplina. A la fecha, la AGP sigue vigente y ha organizado cuatro congresos.

Seis años después, según consta en acta notarial protocolizada de constitución del Colegio Profesional de Psicólogos de Guatemala de fecha 22 de junio de 2005, la Junta Directiva de la AGP, período 2002-2004, decide incluir dentro de su plan de trabajo, la constitución y registro del Colegio de Psicólogos de Guatemala, cuerpo colegiado que agremiaría a los profesionales de las ciencias psicológicas nacionales y extranjeros que ejerzan la profesión en Guatemala. La Junta Directiva Provisional electa en asamblea general estuvo integrada por la maestra Guisela Cárcamo Duarte, presidenta; licenciado César López Gómez, vicepresidente; licenciada María Elena Vargas, secretaria; maestra Sara Pereira Gordillo; tesorera, licenciada Nuvia Leiva Girón, prosecretaria; maestra Ana Cecilia Escobar Martínez, Vocal I; y, maestra Ana María Jurado, Vocal II.

Después de cuatro años de trabajo, el 25 de junio de 2007, la Asamblea de Presidentes de los Colegios Profesionales, aprueba la constitución y registro del Colegio de Psicólogos de Guatemala, según resolución No. 1288.6.07, REF. APCOP 1195.3.03, publicada en el Diario de Centroamérica, diario oficial de Guatemala, el 12 de julio del mismo año.

Tanto la Constitución Política de la República de Guatemala, como la Ley de Colegiación Profesional Obligatoria (Decreto 72-2001), define a los Colegios Profesionales, como asociaciones gremiales no lucrativas, esencialmente apolíticas, de carácter laico, con personalidad jurídica y patrimonio propio.

Los Colegios Profesionales se integran por la Asamblea General, órgano superior y se establecen con la reunión de sus miembros activo. La Junta Directiva que es el órgano ejecutivo, se integra por siete miembro; el Tribunal de Honor que tiene como función primordial conocer de las denuncias, instruir la averiguación y dictar la resolución, imponiendo las sanciones cuando proceda, está compuesto por nueve miembros; y el Tribunal Electoral, órgano conformado por siete miembros, es el órgano superior de los colegios en materia

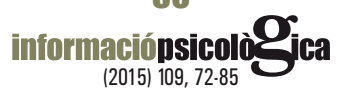


electoral y su función no está supeditada a otro órgano. Funcionan de conformidad con la Ley de Colegiación Profesional Obligatoria (Decreto 72-2001), de su propio estatuto y reglamentos.

La junta directiva provisional logró cumplir con los requisitos establecidos para poder funcionar legalmente, inició con la inscripción de agremiados y en asamblea general eligió al primer tribunal electoral y aprobó su reglamento, el que permitiría convocar a elecciones de junta directiva y tribunal de honor período 2009-2011, la incorporación de su presidenta como miembro de la Asamblea de Presidentes de los Colegios Profesionales, según Punto 12.3, Acta 1291.23/07 sesión ordinaria Asamblea de Presidentes de los Colegios Profesionales, del 23 de julio de 2007, cuerpo colegiado con personería jurídica y patrimonio propio y que dentro de sus funciones principalmente se ocupa de velar y propiciar porque los colegios profesionales cumplan con sus funciones y atribuciones.

El tribunal electoral, tuvo la responsabilidad de convocar al primer proceso electoral para elegir junta directiva y tribunal de honor. El primer tribunal electoral, estuvo presidido por la maestra Ninette Alburez de von Ahn.

El tribunal electoral, convocó a elecciones, celebrándose el acto electoral, en el mes de noviembre de 2008. Elecciones que dieron lugar, para que el 16 de enero de 2009, en acto solemne, tomaran posesión la junta directiva y el tribunal de honor para el período 20092011. La presidenta electa de la junta directiva fue la maestra Guisela Cárcamo Duarte y del tribunal de honor la maestra Ana María Jurado.

En julio de 2010, el colegio fue inscrito como miembro de la Federación Iberoamericana de Psicología, FIAP lográndose también la designación para que Guatemala sea la sede de su X congreso en 2016, según consta en los puntos dos y cuatro del acta de la asamblea general de la FIAP, realizada en Oviedo, España, firmándose además un convenio de cooperación con el Colegio de Psicólogos de España.

El primer tribunal de honor se planteó como prioridad de trabajo la elaboración del Código de Ética del Psicólogo de Guatemala. La construcción del código fue un proceso arduo, se realizaron talleres, conferencias y encuestas en la ciudad capital y en el interior del país, así también se elaboraron dos boletines informativos y de sensibilización. Se contó con la asesoría y acompañamiento de la Dra. Andrea Ferrero de la Universidad de San Luis, Argentina y la orientación del Dr. Janel Gauthier. Para su construcción se tomó como base los códigos de ética de Canadá, México y Nueva Zelanda; se revisó la Declaración Universal de los Principios Éticos para Psicólogos: Un Modelo con Sensibilidad Cultural para la Creación y Revisión de un Código de Ética y otros documentos, como los códigos de ética de Chile, Colombia, México, República Dominicana, Costa Rica, El Salvador y Estados Unidos. El Código de Ética es un instrumento que regula la práctica profesional y asegura que los psicólogos se conduzcan con ética, valores y principios. Se aprobó en Asamblea General extraordinaria el 25 de octubre de 2010 (Colegio de Psicólogos de Guatemala, 2011).

El código de ética se fundamentó en la Declaración Universal de Principios Éticos para Psicólogos, por lo que es el primer código en el mundo que nace de esta Declaración. Parte de cuatro principios universales, el respeto por la dignidad de las personas y los pueblos,

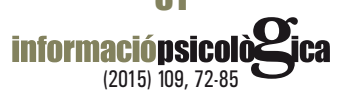


el cuidado responsable del bienestar de los otros, la integridad en las relaciones y la responsabilidad profesional científica con la sociedad. A julio de 2014, el Tribunal de Honor ha conocido nueve casos de denuncia, ha desestimado dos y ha emitido dos sanciones, una privada y una pública.

Desde la constitución del Colegio de Psicólogos de Guatemala, se han realizado tres procesos electorales para elegir junta directiva, tribunal de honor y tribunal electoral. Según consta en el acta No.16-2014 de junta directiva del Colegio de Psicólogos de Guatemala, al 31 de mayo de 2014, el colegio contaba con 5,276 agremiados. Pese a que la Ley de Colegiación Profesional Obligatoria (Decreto 72-2001) en el Artículo 41, Capítulo VII, Disposiciones Finales, Transitorias y Derogatorias, claramente indica que “....no podrá existir más de un Colegio Profesional por cada una de las profesiones universitarias", un buen porcentaje de profesionales no han concretado su traslado al Colegio de Psicólogos de Guatemala, y continúan agremiados en el Colegio Profesional de Humanidades, colegio al que se pertenecía anteriormente. Otros se han graduado, pero debido a que en las empresas o instituciones donde laboran no se les exige la constancia de colegiado activo, no cumplen con colegiarse. Por su parte, según lo norma la ley antes citada, los profesionales que se han graduado en el extranjero deben, para poder colegiarse y ejercer legalmente la profesión en el país, incorporarse a la Universidad de San Carlos, requisito que no todos cumplen.

Por mandato constitucional, los colegios profesionales de Guatemala, se constituyen en cuerpos electorales y participan en la elección a rector de la Universidad de San Carlos. A mayo de 2014, el colegio ha participado en dos de estos procesos.

Actualmente, se cuenta con un colegio profesional que no sólo agremia a los profesionales de las ciencias psicológicas, sino que les da sentido de identidad y pertenencia, que por mandato constitucional les da la potestad legal para ejercer la profesión, promoviendo el desarrollo de la psicología en Guatemala, el desarrollo cultural, científico, profesional y personal de sus agremiados, y sobre todo, velando por el ejercicio ético de la profesión, dignificando así la profesión. El colegio ha llevado a cabo dos congresos nacionales de psicología.

En el esfuerzo por ir construyendo la psicología en Guatemala, así como por la necesidad de adquirir nuevos conocimientos, algunos colegas se han unido para organizar cursos, talleres y diplomados, así como para asistir a congresos en el exterior del país, teniendo una participación activa y reconocida en organizaciones como la Sociedad Interamericana de Psicología. Los congresos regionales de la SIP se inician a propuesta de Guatemala, durante el XXIX congreso de dicha sociedad, llevado a cabo en Lima, Perú, en el año 2003; más tarde, en el año 2004 se realiza el I Congreso Regional en Guatemala y en 2009, se lleva a cabo siempre en Guatemala, el XXXII Congreso de la Sociedad Interamericana de Psicología, bajo la presidencia de la Dra. María del Pilar Grazioso.

El campo de acción del psicólogo en Guatemala se ha ampliado. Según el licenciado Mariano Codoñer Castillo, cada vez más se ha logrado ir incursionando en las distintas esferas de la actividad económica y laboral del país. A su juicio la labor del psicólogo es bien valorada y se requiere constantemente de su participación (M. Codoñer Castillo, comunicación personal, 20 de julio de 2014).

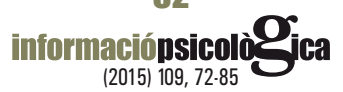


De un actuar tradicional, generalmente desarrollado en la práctica clínica y en el ámbito escolar, la psicología ha ido incursionando y ganando espacios importantes en prácticamente todos los sectores del país. Diferentes procesos y coyunturas, tanto a nivel internacional como nacional pueden haber influido en ello, pero seguramente la firma de la paz en Guatemala en 1996 marcó también un hito en el tiempo que fue además coincidiendo con mayores espacios de participación, resultado del esfuerzo y trabajo de diferentes sectores, así como del reconocimiento de la dignidad humana y de la obligación del Estado de brindar atención y respuesta a las necesidades de su población.

Los psicólogos en Guatemala hoy, realizan un aporte importante al país, no sólo desde la clínica privada, sino también desde el trabajo en instituciones de atención a diferentes grupos poblacionales, servicios de salud, establecimientos educativos y otras instancias de atención social. Además, son parte importante de los procesos de enseñanza, así como en programas de reclutamiento y selección de personal, dirección de recursos humanos y área de mercadeo a nivel empresarial. Participan también, en el diseño y ejecución de programas de tipo social, en el diseño y la planificación de proyectos, pero también en el desarrollo de investigaciones, su aporte desde la academia es valioso para avanzar en el cambio de paradigmas y la desmitificación en la conceptualización de la psicología. Sea en una institución de gobierno o bien desde el poder legislativo y judicial, en una organización no gubernamental, en una instancia de sociedad civil o bien en organismos internacionales, el psicólogo ha ganado un campo de acción y un reconocimiento a nivel social. En área forense, así como de prevención y abordaje de la violencia, problemática que afecta en diferentes dimensiones al país, constituye otra área de relevante aporte. El acompañamiento que psicólogos dan a las familias y víctimas desde diferentes organizaciones, incluyendo la defensa de los derechos humanos y la participación desde las organizaciones basadas en la fe, dan solo una muestra del amplio abanico de acción que constituye la psicología hoy en Guatemala, actividad y aportes que algunas veces pueden ser invisibilizados.

Según el licenciado Mariano Codoñer Castillo (comunicación personal, 20 julio de 2014), en el plano de la psicología organizacional, área del conocimiento que en los últimos años ha tomado auge, no solo por la apertura que a nivel general ha tenido la inversión en el capital humano de instituciones y empresas, sino por el excelente trabajo que algunos profesionales están llevando a cabo no solo en Guatemala, sino en Centro y Sur América y el Caribe, los profesionales de la psicología han incursionado a alto nivel, participando de lleno en el diseño de estrategias y en la toma de decisiones importantes de reconocidas empresas, dejando de lado el rol puramente operativo que en el pasado tenía el psicólogo.

Dentro de los temas que están trabajando, se pueden citar la implementación de estrategias para el incremento de la productividad empresarial, formación de líderes a los más altos niveles de gestión, desarrollo de equipos de alto rendimiento, creación e implementación de modelos para la captación, formación y desarrollo del personal en las organizaciones, creación de sistemas y modelos para gestionar la productividad a través de la mejora del clima organizacional, la evaluación del desempeño y el incremento de la satisfacción del cliente, diseño e implementación de sistemas de gestión organizacional fundamentada en valores, formación de líderes y equipos gerenciales de alto impacto, con fundamento en valores y principios éticos (M. Codoñer Castillo, comunicación personal, 20 de julio de 2014).

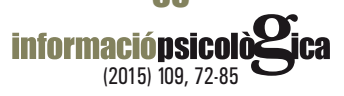


No obstante, la Dra. María del Pilar Grazioso, opina que el papel de la psicología en la regulación, protección y visibilidad de la profesión en especial ante los retos de la sociedad guatemalteca y el Estado, ha sido limitada; sin embargo considera que éste es fundamental, dado que el comportamiento humano constituye la esencia de las ciencias psicológicas (comunicación personal, 18 de julio de 2014). La maestra Ana María Jurado considera que la injerencia de los profesionales de la psicología ha sido modesta en los procesos de prevención, abordaje, intervención y desarrollo, enfocados al bienestar de las personas, aunque si bien es cierto ha sido limitada, la existencia del Colegio de Psicólogos de Guatemala la ha fortalecido (A. Jurado, comunicación personal, 6 de julio de 2014).

Pese a los logros y avances en el campo de la psicología y en el posicionamiento de algunos profesionales de las ciencias psicológicas en el mercado laboral y la actividad económica del país, hay importantes retos por cumplir, especialmente en el campo de la investigación y la generación de conocimiento. Es imprescindible realizar acciones para mejorar la calidad de la formación, propiciar la homologación de las carreras en las universidades, promover la incidencia del psicólogo en el quehacer público y proyectarse a los sectores en condición de mayor vulnerabilidad. La psicología en Guatemala tiene la posibilidad de seguir evolucionando y avanzando, en este proceso el Colegio de Psicólogos, el sector académico y cada profesional tiene un rol que cumplir.

\section{Referencias}

Aguilar G., Recinos, L. Historia y Estado Actual de la Psicología en Guatemala. Revista Latinoamericana de Psicología Volumen 28, Número 2, 1996, pp 197-232. Recuperado de www.redalyc.org/ articulo.oa? id = 80528202

Centro de Investigaciones de las Ciencias de la Salud (2010). Encuesta Nacional de Salud Mental, Resumen Ejecutivo. Guatemala: Universidad de San Carlos de Guatemala.

Colegio de Psicólogos de Guatemala (2007). Estatutos del Colegio de Psicólogos de Guatemala. Ciudad de Guatemala:Tipografía Nacional.

Colegio de Psicólogos de Guatemala (2011) Código de Ética. Ciudad de Guatemala: Servisa Litografía.

Departamento de Psicología. (1 de agosto de 2014). Recuperado de http://psicologia.ufm.edu/

Escuela de Liderazgo y Empresarialidad. (2 de agosto de 2014). Recuperado de http://uspg.edu.gt/webpage/index.php?option $=$ com content \&view $=$ article\&id $=101 \&$ Itemid $=246$

Escuela de Psicopedagogía y Educación. (2 de agosto de 2014). Recuperado de http://uni.edu.gt/index.php/ua/ fhu/epe/

Escuela Superior de Postgrado de Dinámica Humana y Salud mental. (3 de agosto de 2014). Recuperado de http:// www.dinamicahumana.com/

Facultad de Ciencias de la Salud. (3 de agosto de 2014). Recuperado de http://udv.edu.gt/ciencias-de-la-salud/

Facultad de Ciencias Psicológicas. (1 de agosto de 2014). Recuperado de http://www.upana.edu.gt/facultades/ psicologia/

Gobierno de Guatemala, Acuerdo Gubernativo Número 1385-90 de fecha 28 de diciembre de 1990.

Instituto de Ciencias de la Familia. (2 de agosto de 2014). Recuperado de http://www.galileo.edu/icf/ 


\section{dossier: Psicología lberoamericana - ||}

M.Sc Alma Guisela Cárcamo Duarte y M.A Ana Cecilia Escobar Martínez

Ley de Colegiación Profesional Obligatoria, Decreto Número 72-2001. Diario de Centroamérica. Ciudad de Guatemala, 21 de diciembre de 2001.

MSPAS (2008a). Plan Estratégico Nacional para el Fomento de la Salud Mental en Guatemala, 2007-2020. Guatemala: Ministerio de Salud Pública y Asistencia Social.

MSPAS (2008b). Política Nacional de Salud Mental, 2007-2015. Ciudad de Guatemala: Magna Terra Editores, S.A.

Organización Panamericana de la Salud/Organización Mundial de la Salud (2011). Informe del sistema de salud mental en Guatemala. Ciudad de Guatemala: Autor. 